\title{
ON THE NUMBER OF PRIMES LESS THAN OR EQUAL $x$
}

\section{HAROLD N. SHAPIRO}

In the beginnings of the theory of the distribution of prime numbers the unique factorization of $x$ ! is found to be

$$
x !=\prod_{p \leqq x} p^{\alpha_{p}}
$$

where

$$
\alpha_{p}=\left[\frac{x}{p}\right]+\left[\frac{x}{p^{2}}\right]+\cdots .
$$

Taking the logarithm of both sides of (1) and using the very elementary result

$$
\log x !=x \log x+O(x)
$$

there results (see $[1, \text { pp. 72, 98]) })^{1}$

$$
\sum_{p \leqq x}\left[\frac{x}{p}\right] \log p=x \log x+O(x) .
$$

Since $[x / p]=[[x] / p]$, it is clear that (3) then holds for all real $x>0$.

We propose to show in this note that the order of $\pi(x)=$ the number of primes less than or equal $x$ (a result originally due to Tschebyschef, cf. [1]) may be derived very quickly from (3) as a consequence of a general theorem which has no particular relationship to prime numbers. This is of some further interest in that Landau, in $[1, p$. 82 ], says effectively that (3) does not suffice to give Tschebyschef's theorem. Our general theorem, which is in the nature of a weak Tauberian theorem, is as follows.

THEOREM. Let $a_{n} \geqq 0$ be a sequence of real numbers such that

$$
A(x)=\sum_{n \leqq x}\left[\frac{x}{n}\right] a_{n}=x \log x+O(x) .
$$

Then for $S(x)=\sum_{n \leqq x} a_{n}$ there exist two positive constants $\alpha, \beta$, such that for all sufficiently large $x$

$$
\beta x \geqq S(x) \geqq \alpha x .
$$

Proof. Since $[z]-2[z / 2] \geqq 0$ for all positive $z$, we have from (4)

Received by the editors February 22, 1949 and, in revised form, April 3, 1949.

1 Numbers in brackets refer to the reference cited at the end of the paper. 


$$
\begin{aligned}
O(x) & =A(x)-2 A\left(\frac{x}{2}\right)=\sum_{n \leqq x}\left\{\left[\frac{x}{n}\right]-2\left[\frac{x}{2 n}\right]\right\} a_{n} \\
& \geqq \sum_{x / 2<n \leqq x}\left[\frac{x}{n}\right] a_{n}=\sum_{x / 2<n \leqq x} a_{n}=S(x)-S\left(\frac{x}{2}\right) .
\end{aligned}
$$

Thus for some positive $K_{1}$ we have $0 \leqq S(x)-S(x / 2)<K_{1} x$. Then $0 \leqq S\left(x / 2^{i}\right)-S\left(x / 2^{i+1}\right)<K_{1} x / 2^{i}$, from which we obtain via addition $S(x)<2 K_{1} x$. Taking $\beta=2 K_{1}$ yields the upper bound in (5).

Having proved that $S(x)=O(x)$ we return to (4) and obtain

$$
\begin{aligned}
\sum_{n \leqq x}\left[\frac{x}{n}\right] a_{n} & =\sum_{n \leqq x} a_{n}\left\{\frac{x}{n}+O(1)\right\}=x \sum_{n \leqq x} \frac{a_{n}}{n}+O(S(x)) \\
& =x \log x+O(x)
\end{aligned}
$$

whence

$$
T(x)=\sum_{n \leqq x} \frac{a_{n}}{n}=\log x+O(1) .
$$

Thus we can write $T(x)=\log x+R(x)$ where $|R(x)| \leqq K_{2}$ for all sufficiently large $x$. Choosing $\alpha=e^{-2 K_{2}-1}$, we have

$$
T(x)-T(\alpha x)=\sum_{\alpha x<n \leqq x} \frac{a_{n}}{n}=\log \frac{1}{\alpha}+R(x)-R(\alpha x) .
$$

From this we obtain

$$
\frac{1}{\alpha x} S(x) \geqq \frac{1}{\alpha x} \sum_{\alpha x<n \leqq x} a_{n} \geqq \log \frac{1}{\alpha}-2 K_{2}=1
$$

or

$$
S(x) \geqq \alpha x,
$$

as desired. This completes the proof of the theorem.

In order to apply the above theorem to (3) we take

$$
a_{n}=\left\{\begin{array}{cl}
\log n & \text { if } n \text { is a prime, } \\
0 & \text { otherwise, }
\end{array}\right.
$$

so that (4) reduces to (3). Then the theorem gives that for $\theta(x)$ $=\sum_{p \leqq x} \log p$, we have two positive constants $\alpha, \beta$, such that

$$
\beta x \geqq \theta(x) \geqq \alpha x \text {. }
$$

From the obvious inequalities 


$$
\left\{\pi(x)-\pi\left(x^{1 / 2}\right)\right\} \log x^{1 / 2} \leqq \theta(x) \leqq \pi(x) \log x,
$$

Tschebyschef's theorem

$$
\alpha \frac{x}{\log x} \leqq \pi(x) \leqq \gamma \frac{x}{\log x}
$$

now follows at once.

\section{REFERENCE}

1. E. Landau, Handbuch der Lehre von der Verteilung der Primzahlen, vol. 1.

NEW YoRK UNIVERSITY

\section{THE RADICAL OF A NON-ASSOCIATIVE RING}

W. E. JENNER

In this paper a definition is proposed for the radical of a non-associative ring. Our results are somewhat similar to those given for algebras by Albert in [3], ${ }^{1}$ but the difficulties that arose in the earlier theory from absolute divisors of zero have been overcome. With slight modifications, the present proofs are applicable to algebras.

A non-associative ring $\Re$ is an additive abelian group closed under a product operation with respect to which the two distributive laws hold. Multiplication on the right (left) by a fixed element $x \in \Re$ determines an endomorphism $R_{x}\left(L_{x}\right)$ of $\Re$ as an additive group. For $x, y \in \Re$,

$$
x \cdot y=x R_{y}=y L_{x} .
$$

The $R_{x}$ and $L_{y}$ generate an associative ring $\mathfrak{A}$ called the transformation ring of $\Re$. Clearly $\Re$ can be construed as a representation space for $\mathfrak{A}$, and this representation is faithful. The two-sided ideals of $\mathfrak{R}$, which are defined as for associative rings, are exactly the $\mathscr{\Re}$-subspaces of $\Re$. The theory of ring homomorphisms goes over intact to the non-associative case.

A nonzero element $a \in \Re$ is called an absolute divisor of zero if $a \cdot x=x \cdot a=0$ for all $x \in \mathfrak{R}$. If $\mathfrak{A}$ has a unit element $I$ and $\mathfrak{R}$ contains no absolute divisors of zero, then the unit element $I$ is the identity

Received by the editors March 19, 1949.

${ }^{1}$ Numbers in brackets refer to the references cited at the end of the paper. 